PROCEEDINGS OF THE

AMERICAN MATHEMATICAL SOCIETY

Volume 138, Number 2, February 2010, Pages 435-444

S 0002-9939(09)10177-6

Article electronically published on October 5, 2009

\title{
MOTIVES OF HYPERSURFACES OF VERY SMALL DEGREE
}

\author{
ANDRE CHATZISTAMATIOU \\ (Communicated by Ted Chinburg)
}

\begin{abstract}
We study the Chow motive (with rational coefficients) of a hypersurface $X$ in the projective space by using the variety $F(X)$ of $l$-dimensional planes contained in $X$. If the degree of $X$ is sufficiently small, we show that the primitive part of the motive of $X$ is the tensor product of a direct summand in the motive of a suitable complete intersection in $F(X)$ and the $l$-th twist $\mathbb{Q}(-l)$ of the Lefschetz motive.
\end{abstract}

\section{INTRODUCTION}

Let $X$ be a smooth hypersurface of degree $d$ in the projective space $\mathbb{P}_{k}^{n}$ over a field $k$. In this paper we study the Chow motive (with rational coefficients) of $X$ provided that $d$ is sufficiently small.

Roitman has shown that the Chow group of zero-dimensional cycles of degree 0 is a torsion group if $d \leq n$ [4]. For higher dimensional cycles it is known [1, Theorem 4.6] that the Chow groups satisfy

$$
\mathrm{CH}_{l^{\prime}}(X) \otimes \mathbb{Q}=\mathrm{CH}_{l^{\prime}}\left(\mathbb{P}^{n}\right) \otimes \mathbb{Q}=\mathbb{Q} \quad \text { for } 0 \leq l^{\prime} \leq l-1
$$

if $n \geq\left(\begin{array}{c}d+l-1 \\ l\end{array}\right)$ and $d \geq 3$. The identity also holds if $X$ is covered by $l$-dimensional planes [5, Theorem 9.28], or more generally if $X$ is a hyperplane section of a hypersurface $Y$ which is covered by $l$-dimensional planes [3].

Results on triviality of Chow groups give rise to a decomposition of the motive $h(X)$ associated with $X$. In our case, we get

$$
h(X) \cong M_{D} \otimes \mathbb{Q}(-l) \oplus \bigoplus_{i=0}^{l-1} \mathbb{Q}(-i),
$$

where $\mathbb{Q}(-1)$ is the Lefschetz motive and $M_{D}$ is a direct summand of the motive $h(D)$ of some variety $D$. Our purpose is to describe $M_{D}$.

In order to state the theorem we need the following notation. An $l$-dimensional plane $E$ in $\mathbb{P}^{n}$ is called an osculating plane if the intersection $E \cap X$ is an $(l-1)$ dimensional plane or if $E$ is contained in $X$. We say that $X$ has sufficiently many osculating planes if there exists an osculating plane through every closed point of $X$ (the planes may be defined over a field extension of $k$ ).

Received by the editors January 18, 2008, and, in revised form, April 27, 2009.

2000 Mathematics Subject Classification. Primary 14-XX.

The author was supported by a fellowship within the Post-Doc program of the Deutsche Forschungsgemeinschaft (DFG). 
Theorem (see Theorem 2.5). Let n, $d, l$ be numbers such that a general hypersurface of degree $d$ in the projective space $\mathbb{P}^{n}$ has sufficiently many osculating l-dimensional planes. Let $X \subset \mathbb{P}^{n}$ be a smooth hypersurface of degree $d$ such that the Fano variety $F_{l}(X)$ of $l$-dimensional planes contained in $X$ is smooth and has the expected dimension. Furthermore, let $H F_{l}(X) \subset F_{l}(X)$ be a smooth complete intersection of hyperplanes (in the Plücker embedding) with $\operatorname{dim} H F_{l}(X)=n-2 l-1$. Then there is an isomorphism in the category of Chow motives with rational coefficients:

$$
h(X) \cong M_{H F_{l}(X)} \otimes \mathbb{Q}(-l) \oplus \bigoplus_{i=0}^{n-1} \mathbb{Q}(-i),
$$

where $M_{H F_{l}(X)}$ is a direct summand in the motive of $H F_{l}(X)$.

The conditions on $n, d, l$ hold if $n \geq\left(\begin{array}{c}l+d-1 \\ l\end{array}\right)+l-1$. For a finite field $k$ there may be no smooth complete intersection $H F_{l}(X)$ of hyperplanes over $k$. In this case the variety $H F_{l}(X)$ exists over a suitable finite field extension of $k$.

Let us sketch the idea of the proof. We consider the family of planes

$$
\Xi=\left\{(x, E) \in X \times H F_{l}(X) \mid x \in E\right\} \subset X \times H F_{l}(X)
$$

over $H F_{l}(X)$. The cycle $\Xi$ defines a correspondence $\phi_{1}: H F_{l}(X) \otimes \mathbb{Q}(-l) \rightarrow X$, resp. $\phi_{2}: X \rightarrow H F_{l}(X) \otimes \mathbb{Q}(-l)$. The composite $\phi_{1} \circ \phi_{2}$ is the cycle

$$
Z_{X}=\left\{(x, y) \in X \times X \mid x, y \in E, E \in H F_{l}(X)\right\}
$$

in $\mathrm{CH}^{n-1}(X \times X)$. The most important step is to show that

$$
Z_{X}=\imath^{*}(a)+m \cdot \Delta_{X}
$$

for some $a \in \mathrm{CH}^{n-1}\left(\mathbb{P}^{n} \times \mathbb{P}^{n}\right)$, some nonzero integer $m$, the inclusion $\imath: X^{2} \hookrightarrow\left(\mathbb{P}^{n}\right)^{2}$ and the diagonal $\Delta_{X}$. In order to prove (0.0.1) we introduce the doubled incidence variety of degree $d$ hypersurfaces together with two points:

$$
\Sigma=\left\{(x, y, Y) \in\left(\mathbb{P}^{n}\right)^{2} \times \mathbb{P}\left(\operatorname{Sym}^{d}\left(k^{n+1}\right)\right) \mid x, y \in Y\right\} .
$$

The projection $p: \Sigma \rightarrow\left(\mathbb{P}^{n}\right)^{2}$ is a projective bundle over $\left(\mathbb{P}^{n}\right)^{2}-\Delta_{\mathbb{P}^{n}}$ and $\Delta_{\mathbb{P}^{n}}$, so that $\mathrm{CH}^{n-1}(\Sigma)$ can be calculated by using the projective bundle formula and the localization sequence. The cycle $p^{-1}\left(\Delta_{\mathbb{P}^{n}}\right) \in \mathrm{CH}^{n-1}(\Sigma)$ maps to the diagonal $\Delta_{X}$ by the pullback map $\jmath^{*}$ of the inclusion $\jmath: X \times X \rightarrow \Sigma$. One defines a cycle $Z \in \mathrm{CH}^{n-1}(\Sigma)$ with $\jmath^{*}(Z)=Z_{X}$, and by comparing $Z$ with $p^{-1}\left(\Delta_{\mathbb{P}^{n}}\right)$ we obtain (0.0.1) after applying $\jmath^{*}$.

\section{Cycles in the Doubled incidence VARIETy}

1.1. Let $k$ be a field and let $\mathbb{P}\left(\operatorname{Sym}^{d}\left(k^{n+1}\right)\right)$ denote the hypersurfaces of degree $d$ in the projective space $\mathbb{P}_{k}^{n}$. We denote by $\Sigma$ the doubled incidence variety

$$
\Sigma=\left\{(x, y, X) \in\left(\mathbb{P}^{n}\right)^{2} \times \mathbb{P}\left(\operatorname{Sym}^{d}\left(k^{n+1}\right)\right) \mid x, y \in X\right\} .
$$

Letting $p: \Sigma \rightarrow\left(\mathbb{P}^{n}\right)^{2}$ be the projection, we define $\Sigma_{1}:=p^{-1}\left(\Delta_{\mathbb{P}^{n}}\right)$ and $\Sigma_{0}:=$ $\Sigma-\Sigma_{1}$. In the diagram

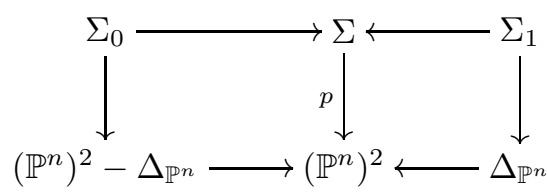


the varieties $\Sigma_{0}$, resp. $\Sigma_{1}$, are projective bundles with fiber dimension $N-2$, resp. $N-1$, where $N=\operatorname{dim} \mathbb{P}\left(\operatorname{Sym}^{d}\left(k^{n+1}\right)\right)$. It is easy to see that $\operatorname{Sing}(\Sigma)=$ $\{(x, x, X) \mid x \in \operatorname{Sing}(X)\}$. The singular locus is a projective bundle over $\Delta_{\mathbb{P}^{n}}$ with fiber dimension $N-1-n$.

1.2. There is an exact sequence

$$
\mathrm{CH}^{0}\left(\Sigma_{1}\right) \rightarrow \mathrm{CH}^{n-1}(\Sigma) \rightarrow \mathrm{CH}^{n-1}\left(\Sigma_{0}\right) \rightarrow 0
$$

from the localization sequence of Chow groups $\left(\mathrm{CH}^{i}\right.$ denotes the group of $i$ codimensional cycles modulo rational equivalence). Moreover, there is a natural splitting defined as follows.

Let $\phi: \Sigma \rightarrow \mathbb{P}\left(\operatorname{Sym}^{d}\left(k^{n+1}\right)\right)$ be the projection and set $c=\phi^{*}\left(c_{1}(\mathcal{O}(1))\right)$. For the other projection $p: \Sigma \rightarrow\left(\mathbb{P}^{n}\right)^{2}$ we may define $p^{*}$ to be the composite $\epsilon^{*} \circ \mathrm{pr}^{*}$, where $\epsilon: \Sigma \rightarrow\left(\mathbb{P}^{n}\right)^{2} \times \mathbb{P}\left(\operatorname{Sym}^{d}\left(k^{n+1}\right)\right)$ is the regular embedding and pr is the projection to $\left(\mathbb{P}^{n}\right)^{2}$. From the projective bundle formula we see that

$$
\bigoplus_{i=0}^{n-1} c^{n-1-i} \cdot p^{*} \mathrm{CH}^{i}\left(\left(\mathbb{P}^{n}\right)^{2}\right) \subset \mathrm{CH}^{n-1}(\Sigma)
$$

splits the sequence (1.2.1), so that every class $Z$ in $\mathrm{CH}^{n-1}(\Sigma)$ can be written as

$$
Z=\sum_{i=0}^{n-1} c^{n-1-i} \cdot p^{*}\left(a_{i}\right)+m \cdot\left[\Sigma_{1}\right]
$$

with $a_{i} \in \mathrm{CH}^{i}\left(\left(\mathbb{P}^{n}\right)^{2}\right)$ and $m$ is an integer.

1.3. Let $\mathcal{H}$ be a smooth, connected projective $k$-scheme and $\Xi \subset \mathcal{H} \times \mathbb{P}^{n}$ be a family of $\kappa$-dimensional subschemes of $\mathbb{P}^{n}$, flat over $\mathcal{H}$. We assume that $\kappa \geq 1$ and that the sheaf $\operatorname{pr}_{1 *}\left(\mathcal{O}_{\Xi} \otimes \operatorname{pr}_{2}^{*} \mathcal{O}_{\mathbb{P}^{n}}(d)\right)$ on $\mathcal{H}$ is locally free. Furthermore, we assume that the map

$$
\operatorname{Sym}^{d}\left(\left(k^{n+1}\right)^{\vee}\right) \otimes \mathcal{O}_{\mathcal{H}}=\operatorname{pr}_{1 *} \operatorname{pr}_{2}^{*} \mathcal{O}_{\mathbb{P}^{n}}(d) \rightarrow \operatorname{pr}_{1 *}\left(\mathcal{O}_{\Xi} \otimes \operatorname{pr}_{2}^{*} \mathcal{O}_{\mathbb{P}^{n}}(d)\right),
$$

induced by $\mathcal{O}_{\mathcal{H} \times \mathbb{P}^{n}} \rightarrow \mathcal{O}_{\Xi}$ and $H^{0}\left(\mathbb{P}^{n}, \mathcal{O}_{\mathbb{P}^{n}}(d)\right)=\operatorname{Sym}^{d}\left(\left(k^{n+1}\right)^{\vee}\right)$, is surjective.

We denote by $E$ the kernel of (1.3.1). It is convenient to write $Q E$ for the sheaf $\operatorname{pr}_{1 *}\left(\mathcal{O}_{\Xi} \otimes \operatorname{pr}_{2}^{*} \mathcal{O}_{\mathbb{P}^{n}}(d)\right)$. In the following commutative diagram we fix the notation for the various maps:

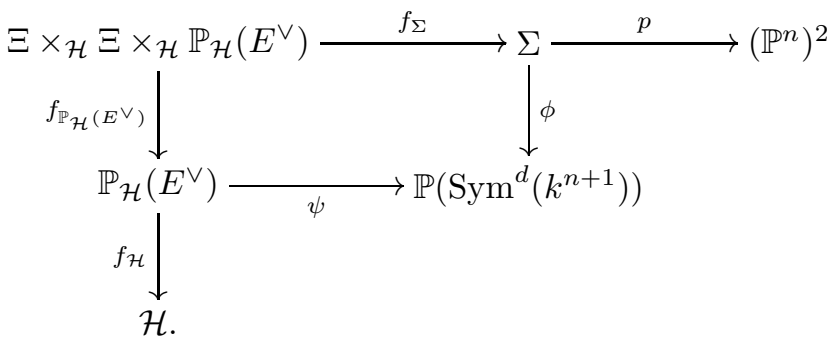

Here $\psi: P_{\mathcal{H}}\left(E^{\vee}\right) \rightarrow \mathbb{P}\left(\operatorname{Sym}^{d}\left(k^{n+1}\right)\right)$ is the composite

$$
\mathbb{P}_{\mathcal{H}}\left(E^{\vee}\right) \stackrel{\tilde{\psi}}{\longrightarrow} \mathcal{H} \times \mathbb{P}\left(\operatorname{Sym}^{d}\left(\left(k^{n+1}\right)^{\vee}\right)\right) \stackrel{\mathrm{pr}_{2}}{\longrightarrow} \mathbb{P}\left(\operatorname{Sym}^{d}\left(\left(k^{n+1}\right)^{\vee}\right)\right),
$$


and $\tilde{\psi}$ is induced by the surjective map $\operatorname{Sym}^{d}\left(\left(k^{n+1}\right)^{\vee}\right) \otimes \mathcal{O}_{\mathcal{H}} \rightarrow E^{\vee}$. By definition, $\mathbb{P}_{\mathcal{H}}\left(E^{\vee}\right)$ is the incidence variety $\left\{(L, X) \in \mathcal{H} \times \mathbb{P}\left(\operatorname{Sym}^{d}\left(\left(k^{n+1}\right)^{\vee}\right)\right) \mid L \subset X\right\}$. The map $f_{\Sigma}$ is defined as follows. By using $\Xi \subset \mathcal{H} \times \mathbb{P}^{n}$ we get

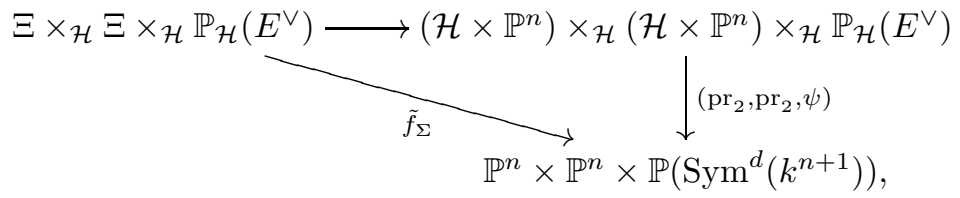

and the image of $\tilde{f}_{\Sigma}$ is the incidence variety $\{(x, y, X) \mid \exists L \in \mathcal{H} x, y \in L \subset X\}$. In particular, $\tilde{f}_{\Sigma}$ factors through $\Sigma$, and we denote this map by $f_{\Sigma}$.

Defining

$$
Z:=f_{\Sigma *} f_{\mathbb{P}_{\mathcal{H}}\left(E^{\vee}\right)}^{*} f_{\mathcal{H}}^{*}: \mathrm{CH}^{*-e}(\mathcal{H}) \rightarrow \mathrm{CH}^{*}(\Sigma),
$$

we will be mainly interested in cycles $Z(a) \in \mathrm{CH}^{n-1}(\Sigma)$ and their pullback to $X \times X \subset \Sigma$ for a hypersurface $X$.

The cycle $\Xi \in \mathrm{CH}^{n-\kappa}\left(\mathcal{H} \times \mathbb{P}^{n}\right)$ has a unique representation,

$$
[\Xi]=\sum_{i=0}^{n-\kappa} \xi_{n-\kappa-i} \otimes H^{i}
$$

with $\xi_{j} \in \mathrm{CH}^{j}(\mathcal{H})$ and $H=c_{1}\left(\mathcal{O}_{\mathbb{P}^{n}}(1)\right)$.

Lemma 1.4. For $a \in \mathrm{CH}^{n-1-e}(\mathcal{H})$, let $Z(a)_{\mid \Sigma_{0}}=\sum_{i=0}^{n-1} c^{n-1-i} \cdot p^{*}\left(a_{i}\right)$ be the pullback of $Z(a)$ to $\Sigma_{0}$. The classes $a_{i}$ can be computed as follows:

$$
\sum_{i} a_{i}=\frac{\sum_{0 \leq s, t \leq n-\kappa} b_{s, t} H^{s} \otimes H^{t}}{(1+d \otimes H)(1+H \otimes d)}
$$

in $\mathrm{CH}^{*}\left(\left(\mathbb{P}^{n}\right)^{2}-\Delta_{\mathbb{P}^{n}}\right)$ and where

$$
b_{s, t}=\int_{\mathcal{H}} \xi_{n-\kappa-s} \cdot \xi_{n-\kappa-t} \cdot c_{\mathrm{rk}(Q E)-n+s+t-1}(Q E) \cdot a .
$$

Proof. On $U:=\left(\mathbb{P}^{n}\right)^{2}-\Delta_{\mathbb{P}^{n}}$ the evaluation morphism

$$
\operatorname{Sym}^{d}\left(\left(k^{n+1}\right)^{\vee}\right) \otimes \mathcal{O}_{U} \rightarrow \operatorname{pr}_{1}^{*} \mathcal{O}_{\mathbb{P}^{n}}(d) \oplus \operatorname{pr}_{2}^{*} \mathcal{O}_{\mathbb{P}^{n}}(d)
$$

is surjective; let $G$ be the kernel. We have $\Sigma_{0}=\mathbb{P}_{U}\left(G^{\vee}\right)$ and $c_{\mid \Sigma_{0}}=c_{1}\left(\mathcal{O}_{\mathbb{P}_{U}\left(G^{\vee}\right)}(1)\right)$, so that

$$
p_{*}\left(\frac{1}{1-c} Z(a)_{\mid \Sigma_{0}}\right)=\sum_{j \geq 0} s_{j}(G) \cdot \sum_{i=0}^{n-1} a_{i},
$$

where $s_{j}(G)$ are the Segre classes. Since $G$ is the kernel of (1.4.1), we see that

$$
\left(1+d \cdot \operatorname{pr}_{1}^{*} c_{1}\left(\mathcal{O}_{\mathbb{P}^{n}}(1)\right)\right)\left(1+d \cdot \operatorname{pr}_{2}^{*} c_{1}\left(\mathcal{O}_{\mathbb{P}^{n}}(1)\right)\right) \sum_{j \geq 0} s_{j}(G)=1 .
$$

Define

$$
T_{i}(a)=p_{*}\left(c^{N-n-1+i} Z(a)\right)
$$


and let $\jmath: U \subset\left(\mathbb{P}^{n}\right)^{2}$ be the open immersion. It follows from (1.4.2) and (1.4.3) that

$$
\sum_{i=0}^{n-1} a_{i}=\frac{\sum_{i \geq 0} J^{*} T_{i}(a)}{(1+d \otimes H)(1+H \otimes d)} .
$$

Let us now compute the $T_{i}$ :

$$
\begin{aligned}
p_{*}\left(c^{N-n-1+i} Z(a)\right) & =p_{*} f_{\Sigma *}\left(\left(\phi \circ f_{\Sigma}\right)^{*} c_{1}(\mathcal{O}(1))^{N-n-1+i} \cdot\left(f_{\mathcal{H}} \circ f_{\mathbb{P}_{\mathcal{H}}\left(E^{\vee}\right)}\right)^{*}(a)\right) \\
& =p_{*} f_{\Sigma *} f_{\mathbb{P}_{\mathcal{H}}\left(E^{\vee}\right)}^{*}\left(\psi^{*} c_{1}(\mathcal{O}(1))^{N-n-1+i} \cdot f_{\mathcal{H}}^{*}(a)\right) .
\end{aligned}
$$

The map $T=p_{*} f_{\Sigma *} f_{\mathbb{P}_{\mathcal{H}}\left(E^{\vee}\right)}^{*}$ is given by the correspondence $\mathbb{P}_{\mathcal{H}}\left(E^{\vee}\right) \times_{\mathcal{H}} \Xi \times_{\mathcal{H}} \Xi$ in $\mathbb{P}_{\mathcal{H}}\left(E^{\vee}\right) \times\left(\mathbb{P}^{n}\right)^{2}$, and using (1.3.3), we see that

$$
[\Xi \times \mathcal{H} \Xi]=\sum_{0 \leq s, t \leq n-\kappa}\left(\xi_{n-\kappa-s} \cdot \xi_{n-\kappa-t}\right) \otimes H^{s} \otimes H^{t}
$$

in $\mathrm{CH}^{*}\left(\mathcal{H} \times\left(\mathbb{P}^{n}\right)^{2}\right)$, so that the coefficient of $H^{s} \otimes H^{t}$ in $T_{i}(a)$ is

$$
\begin{aligned}
\int_{\mathbb{P}_{\mathcal{H}}\left(E^{\vee}\right)} f_{\mathcal{H}}^{*} \xi_{n-\kappa-s} & \cdot f_{\mathcal{H}}^{*} \xi_{n-\kappa-t} \cdot \psi^{*} c_{1}(\mathcal{O}(1))^{N-n-1+i} \cdot f_{\mathcal{H}}^{*}(a) \\
= & \int_{\mathcal{H}} \xi_{n-\kappa-s} \cdot \xi_{n-\kappa-t} \cdot s_{N-n-\mathrm{rk}(E)+i}(E) \cdot a .
\end{aligned}
$$

If $Z(a) \in \mathrm{CH}^{n-1}(\Sigma)$, then $T_{i}(a) \in \mathrm{CH}^{i}\left(\left(\mathbb{P}^{n}\right)^{2}\right)$ by definition so that the coefficient of $H^{s} \otimes H^{t}$ in $T_{i}(a)$ vanishes if $s+t \neq i$. The identity $s_{N-n-\operatorname{rk}(E)+i}(E)=$ $c_{\mathrm{rk}(Q E)-n-1+i}(Q E)$ completes the proof.

The next lemma computes the pullback of $Z(a)$ to $X \times X$ for a smooth hypersurface $X$. We write $\imath_{\Sigma}$ for the inclusion $X \times X \rightarrow \Sigma$ and $\imath_{\left(\mathbb{P}^{n}\right)^{2}}$ for the inclusion $X \times X \rightarrow\left(\mathbb{P}^{n}\right)^{2}$. Note that both inclusions are locally complete intersections; thus the pullback is well-defined. Indeed, since $X$ is smooth the image $\imath_{\Sigma}(X \times X)$ is contained in the open smooth part of $\Sigma$ (see (1.1)).

Lemma 1.5. For $Z(a) \in \mathrm{CH}^{n-1}(\Sigma)$ and $Z(a)_{\mid \Sigma_{0}}=\sum_{i=0}^{n-1} c^{n-1-i} \cdot p^{*}\left(a_{i}\right)$, we have

$$
\imath_{\Sigma}^{*} Z(a)=\imath_{\left(\mathbb{P}^{n}\right)^{2}}^{*}\left(a_{n-1}\right)-m \cdot \Delta_{X}
$$

where

$$
m=d \cdot \sum_{j=\kappa-1}^{n-1}(-d)^{j} \int_{\mathcal{H}} \xi_{n-\kappa} \cdot \xi_{j-\kappa+1} \cdot c_{\mathrm{rk}(Q E)-2-j}(Q E) \cdot a .
$$

Proof. We know that

$$
Z(a)=\sum_{i=0}^{n-1} c^{n-1-i} \cdot p^{*}\left(a_{i}\right)-m \cdot\left[\Sigma_{1}\right]
$$

for some $m$. The line bundle $\imath_{\Sigma}^{*} \phi^{*} \mathcal{O}(1)$ is trivial and $\imath_{\Sigma}^{*}\left[\Sigma_{1}\right]=\Delta_{X}$; therefore

$$
\imath_{\Sigma}^{*} Z(a)=\imath_{\left(\mathbb{P}^{n}\right)^{2}}^{*} a_{n-1}-m \cdot \Delta_{X} .
$$

We claim that

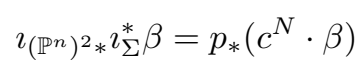

for every class $\beta \in \mathrm{CH}^{*}(\Sigma)$. This follows from the following fact. If $g: D \subset Y$ is a Cartier divisor on $Y$ and $L$ is the associated line bundle, then $g_{*} g^{*}(\beta)=c_{1}(L) \cdot \beta$. 
Claim (1.5.3) implies $\imath_{\left(\mathbb{P}^{n}\right)^{2} *} \imath_{\Sigma}^{*} Z(a)=T_{n+1}(a)$ (see (1.4.4)). The coefficient of $H \otimes H^{n}$ in $T_{n+1}(a)$ is computed in (1.4.6) and vanishes for trivial reasons (if $\kappa \geq 1$ ). Using Lemma 1.4, we see that the coefficient $\gamma$ of $1 \otimes H^{n-1}$ in $a_{n-1}$ is

$$
\gamma=\sum_{j=\kappa-1}^{n-1}(-d)^{j} \cdot b_{0, n-1-j} .
$$

By applying $\imath_{\left(\mathbb{P}^{n}\right)^{2} *}$ to $(1.5 .2)$ and using $\imath_{\left(\mathbb{P}^{n}\right)^{2} *} \imath_{\left(\mathbb{P}^{n}\right)^{2}}^{*} a_{n-1}=d^{2}(H \otimes H) \cdot a_{n-1}$ and $\imath_{\left(\mathbb{P}^{n}\right)^{2} *} \Delta_{X}=d \cdot \sum_{i \geq 0}^{n-1} H^{i+1} \otimes H^{n-i}$, we find that $m=d \cdot \gamma$, as claimed.

\section{Motives of HYPERsurfaces AND THEIR FANO VARIETIES}

2.1. In the following we work with the Grassmannian of $\kappa$-planes $\mathcal{H}=\mathrm{Gr}_{\kappa}$ in projective space where $\Xi \subset \mathrm{Gr}_{\kappa} \times \mathbb{P}^{n}$ is the universal family. We denote by $V$, resp. $Q V$, the tautological bundle $V \subset \mathcal{O}_{\mathrm{Gr}}^{n+1}$, resp. the quotient $\mathcal{O}_{\mathrm{Gr}}^{n+1} / V$. The family $\Xi$ is the projective bundle $\Xi=\mathbb{P}_{\mathrm{Gr}_{\kappa}}\left(V^{\vee}\right)$, and it is easy to see that

$$
[\Xi]=\sum_{i=0}^{n-\kappa} c_{n-\kappa-i}(Q V) \otimes H^{i}
$$

in $\mathrm{CH}^{n-\kappa}\left(\mathrm{Gr} \times \mathbb{P}^{n}\right)$. Furthermore we have that $Q E=\operatorname{pr}_{1 *}\left(\mathcal{O}_{\Xi} \otimes \operatorname{pr}_{2}^{*} \mathcal{O}_{\mathbb{P}^{n}}(d)\right)=$ $\operatorname{Sym}^{d}\left(V^{\vee}\right)$.

2.2. We will be interested in cycles $Z\left(c_{1}\left(V^{\vee}\right)^{s}\right) \in \mathrm{CH}^{n-1}(\Sigma)$, for $s \geq 0$ (notation as in (1.3.2) $)$. By counting dimensions we see that

$$
s=\operatorname{dim} \Xi-\operatorname{rk}(Q E)-(n-1)=\kappa(n-\kappa)-\left(\begin{array}{c}
d+\kappa \\
\kappa
\end{array}\right)+\kappa+1 .
$$

Let us consider the variety

$$
\begin{aligned}
& \left\{\left(x, E_{\kappa-1}, E_{\kappa}, X\right) \in \mathbb{P}^{n} \times \operatorname{Gr}_{\kappa-1} \times \operatorname{Gr}_{\kappa} \times \mathbb{P}\left(\operatorname{Sym}^{d}\left(k^{n+1}\right)\right) \mid\right. \\
& \left.\quad x \in E_{\kappa-1} \subset E_{\kappa}, E_{\kappa} \cap X=E_{\kappa-1} \text { or } E_{\kappa} \subset X\right\} .
\end{aligned}
$$

More formally, this variety is defined as follows. On $\Xi=\mathbb{P}_{\mathrm{Gr}_{\kappa}}\left(V^{\vee}\right)$ there is an exact sequence of vector bundles

$$
0 \rightarrow V_{1}^{\vee} \rightarrow V^{\vee} \otimes \mathcal{O}_{\Xi} \rightarrow \mathcal{O}_{\Xi}(1) \rightarrow 0,
$$

and the points of $\mathbb{P}_{\Xi}\left(V_{1}\right)$ are $\left\{\left(x, E_{\kappa-1}, E_{\kappa}\right) \mid x \in E_{\kappa-1} \subset E_{\kappa}\right\}$.

Since $\mathcal{O}_{\mathbb{P}_{\Xi}\left(V_{1}\right)}(-1) \subset V_{1}^{\vee} \otimes \mathcal{O}_{\mathbb{P}_{\Xi}\left(V_{1}\right)} \subset V^{\vee} \otimes \mathcal{O}_{\mathbb{P}_{\Xi}\left(V_{1}\right)}$, we can define $G$ to be the kernel of

$$
\operatorname{Sym}^{d}\left(\left(k^{n+1}\right)^{\vee}\right) \otimes \mathcal{O}_{\mathbb{P}_{\Xi}\left(V_{1}\right)} \rightarrow \operatorname{Sym}^{d}\left(V^{\vee}\right) / \mathcal{O}_{\mathbb{P}_{\Xi}\left(V_{1}\right)}(-d) .
$$

Then $\mathbb{P}_{\mathbb{P}_{\Xi}\left(V_{1}\right)}\left(G^{\vee}\right)$ is the variety (2.2.2).

The following condition will imply that the diagonal $\Delta_{X}$, for a hypersurface $X$, can be written in terms of the pullback of $Z\left(c_{1}\left(V^{\vee}\right)^{s}\right)$ to $X \times X$ (i.e. $m \neq 0$ in Lemma 1.5).

(B) The following map is surjective:

$$
\begin{aligned}
& \mathbb{P}_{\mathbb{P}_{\Xi}\left(V_{1}\right)}\left(G^{\vee}\right) \rightarrow\left\{(x, X) \in \mathbb{P}^{n} \times \mathbb{P}\left(\operatorname{Sym}^{d}\left(k^{n+1}\right)\right) \mid x \in X\right\}, \\
& \left(x, E_{\kappa-1}, E_{\kappa}, X\right) \mapsto(x, X) .
\end{aligned}
$$

By counting dimensions we see that a necessary condition for (B) is $s \geq 0$ (with $s$ as in (2.2.1) $)$. If $d=2$, then $s \geq 0$ is not sufficient; the first example is $\kappa=3$ 
and $n=5$. In fact, (B) is equivalent to $n \geq 2 \cdot \kappa$ if $d=2$, which can be checked by using the following lemma. However, we don't know any counterexamples to

$$
(B) \Leftrightarrow s \geq 0
$$

for $d>2$. If $\kappa=1$, then (2.2.4) is true. This also holds for

$$
(n, d, \kappa)=(6,3,2),(8,4,2),(11,5,2),(9,3,3) .
$$

It is known [1, Lemma 1.1+Lemma 4.2] that (B) is true if $d \geq 3$ and

$$
n-\kappa+1 \geq\left(\begin{array}{c}
\kappa-1+d \\
\kappa
\end{array}\right) .
$$

Lemma 2.3. Condition (B) holds if and only if

$$
m=d \cdot \sum_{j=\kappa-1}^{n-1}(-d)^{j} \int_{\mathcal{H}} \xi_{n-\kappa} \cdot \xi_{j-\kappa+1} \cdot c_{\mathrm{rk}(Q E)-2-j}(Q E) \cdot c_{1}\left(V^{\vee}\right)^{s}
$$

is nonzero.

Proof. From the construction of $\mathbb{P}_{\mathbb{P}_{\Xi}\left(V_{1}\right)}\left(G^{\vee}\right)$ we have the maps

$$
\mathbb{P}_{\mathbb{P}_{\Xi}\left(V_{1}\right)}\left(G^{\vee}\right) \stackrel{f}{\rightarrow} \mathbb{P}_{\Xi}\left(V_{1}\right) \stackrel{g}{\rightarrow} \Xi \stackrel{h}{\rightarrow} \mathrm{Gr}_{\kappa} .
$$

We claim that

$$
(h \circ g \circ f)_{*}\left(c_{1}\left(\mathcal{O}_{\Xi}(1)\right)^{n-1} \cdot c_{1}\left(\mathcal{O}_{\mathbb{P}_{\mathbb{P}_{\Xi}\left(V_{1}\right)}\left(G^{\vee}\right)}(1)\right)^{N}\right)
$$

$$
=(-1)^{\kappa-1} d \cdot \sum_{j=\kappa-1}^{n-1}(-d)^{j} \xi_{n-\kappa} \cdot \xi_{j-\kappa+1} \cdot c_{\mathrm{rk}(Q E)-2-j}(Q E)
$$

$\left(N=\operatorname{dim} \mathbb{P}\left(\operatorname{Sym}^{d}\left(k^{n+1}\right)\right)\right)$. Indeed,

$g_{*} f_{*}\left(c_{1}\left(\mathcal{O}_{\mathbb{P}_{\mathbb{P}_{\Xi}\left(V_{1}\right)}\left(G^{\vee}\right)}(1)\right)^{N}\right)=g_{*} s_{\mathrm{rk}(Q E)-1}(G)=g_{*} c_{\mathrm{rk}(Q E)-1}\left(Q E / \mathcal{O}_{\mathbb{P}_{\Xi}\left(V_{1}\right)}(-d)\right)=$

$g_{*} \sum_{i} d^{i} c_{\mathrm{rk}(Q E)-i-1}(Q E) \cdot c_{1}\left(\mathcal{O}_{\mathbb{P} \Xi\left(V_{1}\right)}(1)\right)^{i}=\sum_{i} d^{i} c_{\mathrm{rk}(Q E)-i-1}(Q E) \cdot s_{i-\kappa+1}\left(V_{1}^{\vee}\right)$,

and from (2.2.3) we obtain $s_{j}\left(V_{1}^{\vee}\right)=s_{j}\left(V^{\vee}\right)+c_{1}\left(\mathcal{O}_{\Xi}(1)\right) \cdot s_{j-1}\left(V^{\vee}\right)$ for all $j$. Thus,

$$
\begin{aligned}
& (h \circ g \circ f)_{*}\left(c_{1}\left(\mathcal{O}_{\Xi}(1)\right)^{n-1} \cdot c_{1}\left(\mathcal{O}_{\mathbb{P}_{\mathbb{P}_{\Xi}\left(V_{1}\right)}\left(G^{\vee}\right)}(1)\right)^{N}\right) \\
& =\sum_{i} d^{i} c_{\operatorname{rk}(Q E)-i-1}(Q E) \cdot\left(s_{n-\kappa-1}(V) s_{i-\kappa+1}\left(V^{\vee}\right)+s_{n-\kappa}(V) s_{i-\kappa}\left(V^{\vee}\right)\right) .
\end{aligned}
$$

On $\Xi$ the natural morphism $Q E \otimes \mathcal{O}_{\Xi}(-d) \rightarrow \mathcal{O}_{\Xi}$ is surjective, so that the top Chern class of $Q E \otimes \mathcal{O}_{\Xi}(-d)$ vanishes:

$$
\begin{aligned}
& h_{*} c_{\mathrm{rk}(Q E)}\left(Q E \otimes \mathcal{O}_{\Xi}(-d)\right)=h_{*} \sum_{i}(-d)^{i} c_{\mathrm{rk}(Q E)-i}(Q E) \cdot c_{1}\left(\mathcal{O}_{\Xi}(1)\right)^{i} \\
& =\sum_{i}(-d)^{i} c_{\mathrm{rk}(Q E)-i}(Q E) s_{i-\kappa}(V)=(-1)^{\kappa} d \cdot \sum_{i} d^{i} c_{\mathrm{rk}(Q E)-i-1}(Q E) s_{i+1-\kappa}\left(V^{\vee}\right)
\end{aligned}
$$

is zero and together with (2.3.2) we obtain

$$
\begin{aligned}
(h \circ g \circ f)_{*}\left(c_{1}\left(\mathcal{O}_{\Xi}(1)\right)^{n-1} \cdot c_{1}\left(\mathcal{O}_{\mathbb{P}_{\mathbb{P}_{\Xi}\left(V_{1}\right)}\left(G^{\vee}\right)}(1)\right)^{N}\right) \\
=d \cdot \sum_{i} d^{i} c_{\mathrm{rk}(Q E)-i-2}(Q E) \cdot s_{n-\kappa}(V) s_{i+1-\kappa}\left(V^{\vee}\right) .
\end{aligned}
$$

Then, $(-1)^{j} s_{j}\left(V^{\vee}\right)=s_{j}(V)=c_{j}(Q V)=\xi_{j}$ proves the claim. 
Let $\pi$ be the map in condition (B). For a general closed point $(x, X)$ the irreducible components of $\pi^{-1}(x, X)$ map generically one to one to $\mathrm{Gr}_{\kappa}$. The class $c_{1}\left(V^{\vee}\right)=c_{1}\left(\Lambda^{\kappa+1} V^{\vee}\right)$ is the class of an ample line bundle, and $s$ is the dimension of the generic fiber of $\pi$ if $\pi$ is surjective. Thus, $\pi$ is surjective if and only if $\int_{\mathbb{P}_{\mathbb{P}_{\Xi}\left(V_{1}\right)}\left(G^{\vee}\right)} c_{1}\left(V^{\vee}\right)^{s} \cdot\left[\pi^{-1}(x, X)\right]>0$.

$$
\begin{gathered}
\text { Since } \mathcal{O}_{\Xi}(1)=\pi^{*} \mathcal{O}_{\mathbb{P} n}(1) \text { and } \mathcal{O}_{\mathbb{P}_{\mathbb{P}} \Xi\left(V_{1}\right)}\left(G^{\vee}\right) \\
\pi^{*}(z)=c_{1}\left(\mathcal{O}_{\Xi}(1)\right)^{n-1} \cdot c_{1}\left(\mathcal{O}_{\mathbb{P}_{\mathbb{P}_{\Xi}\left(V_{1}\right)}\left(G^{\vee}\right)}(1)\right)^{N}, \\
z=c_{1}\left(\mathcal{O}_{\mathbb{P}^{n}}(1)\right)^{n-1} \cdot c_{1}\left(\mathcal{O}_{\left.\mathbb{P}_{\left(\operatorname{Sym}^{d}\left(k^{n+1}\right)\right)}(1)\right)^{N} .} .\right.
\end{gathered}
$$

The zero-dimensional cycle $z$ is the class of a complete intersection of $n-1$ hyperplanes in $\mathbb{P}^{n}$ and $N$ hyperplanes in $\mathbb{P}\left(\operatorname{Sym}^{d}\left(k^{n+1}\right)\right)$. Therefore $z=\sum_{i}\left(x_{i}, X_{i}\right)$ for some sufficiently general points $\left(x_{i}, X_{i}\right)$, and $c_{1}\left(\mathcal{O}_{\Xi}(1)\right)^{n-1} \cdot c_{1}\left(\mathcal{O}_{\mathbb{P}_{\mathbb{P}_{\Xi}\left(V_{1}\right)}\left(G^{\vee}\right)}(1)\right)^{N}=$ $\sum_{i} \pi^{*}\left(x_{i}, X_{i}\right)$. Now 2.3.1) implies the lemma.

In the following theorem we work with the category of (pure) Chow motives with rational coefficients (see [2, Chapter 16]), where $\mathbb{Q}(-1)$ denotes the Lefschetz motive.

Theorem 2.5. Let $n, d, \kappa$ be numbers satisfying (B). Let $X \subset \mathbb{P}^{n}$ be a smooth hypersurface of degree $d$ such that the Fano variety $F_{\kappa}(X)$ of $\kappa$-dimensional planes contained in $X$ has the expected dimension (i.e. $\left.\operatorname{dim} F_{\kappa}(X)=(\kappa+1)(n-\kappa)-\left(\begin{array}{c}d+\kappa \\ \kappa\end{array}\right)\right)$, and let $H F_{\kappa}(X) \subset F_{\kappa}(X)$ be a complete intersection of hyperplanes (in the Plücker embedding) with $\operatorname{dim} H F_{\kappa}(X)=n-2 \kappa-1$. Furthermore, let $\psi: \widetilde{H F_{\kappa}(X)} \rightarrow$ $H F_{\kappa}(X)$ be a generically finite surjective morphism which resolves the singularities of $H F_{\kappa}(X)$. Then there is an isomorphism in the category of Chow motives with rational coefficients

$$
\left.\left(X, i d_{X}\right) \cong\left(\widehat{H F_{\kappa}(X}\right), P\right) \otimes \mathbb{Q}(-\kappa) \oplus \bigoplus_{i=0}^{n-1} \mathbb{Q}(-i)
$$

for a suitable projector $P$. We give an explicit formula for $P$ in the proof.

Proof. From Lemma 1.5 and Lemma 2.3 we obtain

$$
\Delta_{X}=-\frac{1}{m} \imath_{\Sigma}^{*} Z\left(c_{1}\left(V^{\vee}\right)^{s}\right)+\frac{1}{m} \imath_{\left(\mathbb{P}^{n}\right)^{2}}^{*}\left(a_{n-1}\right) .
$$

In the Cartesian diagram

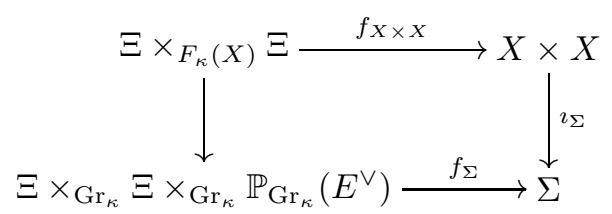

the pullback and the pushforward commute [2, Theorem 6.2, Remark 6.2.1], because $F_{\kappa}(X)$ has the expected dimension. Therefore

$$
\imath_{\Sigma}^{*} Z\left(c_{1}\left(V^{\vee}\right)^{s}\right)=f_{X \times X *}\left(c_{1}\left(V^{\vee}\right)^{s}\right)=\left(f_{X \times X} \circ \eta\right)_{*}\left(\left[\Xi \times_{H F_{\kappa}(X)} \Xi\right]\right),
$$

where $\eta: H F_{\kappa}(X) \rightarrow F_{\kappa}(X)$ is a complete intersection of $s$ hyperplanes (thus the dimension is $\left.\operatorname{dim} H F_{\kappa}(X)=n-2 \kappa-1\right)$. It is more convenient to write $H=H F_{\kappa}(X)$ and $\tilde{H}=\widehat{H F_{\kappa}(X)}$. 
There is a cycle $Y \in \mathrm{CH}_{n-2 \kappa-1}(\tilde{H}) \otimes \mathbb{Q}$ (i.e. $Y$ is a rational linear combination of connected components of $\tilde{H})$ such that $\psi_{*}(Y)=[H]$.

Let $\phi_{1} \in \operatorname{Cor}(\tilde{H} \otimes \mathbb{Q}(-\kappa), X)$ (resp. $\phi_{2} \in \operatorname{Cor}(X, \tilde{H} \otimes \mathbb{Q}(-\kappa))$ ) be the correspondence defined by the cycle $\tilde{H} \times_{H} \Xi$ in $\tilde{H} \times X\left(\operatorname{resp} .\left[\Xi \times_{H} \tilde{H}\right] \cdot p r_{\tilde{H}}^{*}(Y)\right.$ in $X \times \tilde{H}$, where $\operatorname{pr}_{\tilde{H}}: X \times \tilde{H} \rightarrow \tilde{H}$ is the projection).

We consider the commutative diagram

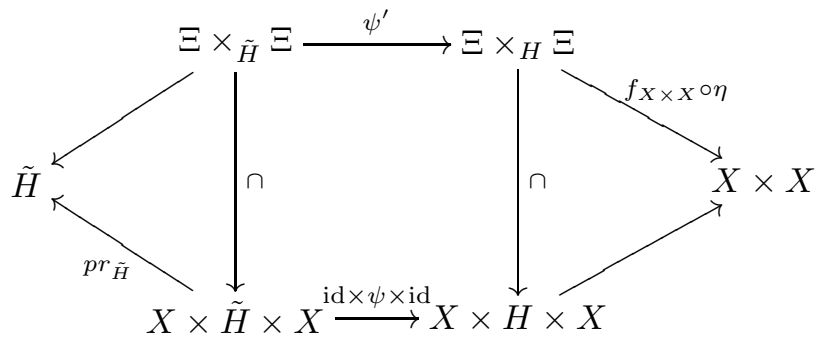

It is easy to see that $\phi_{1} \circ \phi_{2}=\left(f_{X \times X} \circ \eta \circ \psi^{\prime}\right)_{*}\left(\left[\Xi \times_{\tilde{H}} \Xi\right] \cdot p r_{\tilde{H}}^{*}(Y)\right)$, and $\psi_{*}^{\prime}\left(\left[\Xi \times_{\tilde{H}}\right.\right.$ $\left.\Xi] \cdot \operatorname{pr}_{\tilde{H}}^{*}(Y)\right)=\left[\Xi \times_{H} \Xi\right]$ together with $(2.5 .2)$ yields

$$
\phi_{1} \circ \phi_{2}=Z\left(c_{1}\left(V^{\vee}\right)^{s}\right) .
$$

If $H$ is the class of a hyperplane in $\mathbb{P}^{n}$, then we write $P_{i}$ for the pullback of $\frac{1}{d} H^{n-1-i} \otimes H^{i} \in \mathrm{CH}^{n-1}\left(\mathbb{P}^{n} \times \mathbb{P}^{n}\right) \otimes \mathbb{Q}$ to $X \times X$. The correspondences $P_{0}, \ldots, P_{n-1}$ are idempotent and orthogonal. We may write $a_{n-1}=\frac{m}{d} \sum_{i=0}^{n-1} \beta_{i} H^{n-1-i} \otimes H^{i}$, and it follows from (2.5.1) and (2.5.3) that

$$
\Delta_{X}-\sum_{i} P_{i}=-\frac{1}{m} \phi_{1} \circ \phi_{2}+\sum_{i=0}^{n-1}\left(\beta_{i}-1\right) P_{i} .
$$

Composition with $P_{i}$ shows that $\frac{1}{m} \phi_{1} \circ \phi_{2} \circ P_{i}=\left(\beta_{i}-1\right) P_{i}$ and

$$
\Delta_{X}-\sum_{i} P_{i}=\phi_{1} \circ\left(-\frac{1}{m} \phi_{2}+\frac{1}{m} \sum_{i=0}^{n-1} \phi_{2} \circ P_{i}\right) .
$$

Since $\left(X, P_{i}\right) \cong \mathbb{Q}(-i)$ and $\left(X, \Delta_{X}-\sum_{i} P_{i}\right) \cong\left(\tilde{H},-\frac{1}{m} \phi_{2} \circ\left(\Delta_{X}-\sum_{i} P_{i}\right) \circ \phi_{1}\right)$ by (2.5.4), this proves the theorem.

\section{ACKNOWLEDGMENTS}

I thank H. Esnault for introducing me to this subject and for her interest in my work. I am grateful to Y. André for valuable discussions. I thank the École Normale Supérieure for its hospitality.

\section{REFERENCES}

[1] Esnault, Hélène; Levine, Marc; Viehweg, Eckart, Chow groups of projective varieties of very small degree, Duke Math. J. 87 (1997), 29-58. MR1440062 (98d:14002)

[2] Fulton, William, Intersection theory. Second edition. Ergebnisse der Mathematik und ihrer Grenzgebiete. 3. Folge, Springer-Verlag, Berlin, 1998. MR1644323 (99d:14003)

[3] Otwinowska, Anna, Remarques sur les groupes de Chow des hypersurfaces de petit degré [Remarks on Chow groups of hypersurfaces of low degree], C. R. Acad. Sci. Paris Sér. I Math. 329 (1999), 51-56. MR:1703267 (2000g:14007) 
[4] Roŭtman, A. A., Rational equivalence of zero-dimensional cycles. (Russian), Mat. Sb. (N.S.) 89 (131) (1972), 569-585. (Translation in Math. USSR-Sb. 18 (1974), 571-588.) MR 0327767 (48:6109)

[5] Voisin, Claire, Hodge theory and complex algebraic geometry. II. Translated from the French by Leila Schneps. Cambridge Studies in Advanced Mathematics, 77, Cambridge University Press, Cambridge, 2003. MR 1997577 (2005c:32024b)

Fachbereich Mathematik, Universität Duisburg-Essen, 45117 Essen, Germany

E-mail address: a.chatzistamatiou@uni-due.de 